\title{
BLIND NOISE VARIANCE ESTIMATION FOR OFDMA SIGNALS
}

\author{
François-Xavier Socheleau, Dominique Pastor, Abdeldjalil Aïssa-El-Bey, Sébastien Houcke \\ Institut TELECOM; TELECOM Bretagne; UMR CNRS 3192 Lab-STICC, \\ Université européenne de Bretagne, France
}

\begin{abstract}
We present two new noise variance estimation methods for OFDMA signals transmitted through an unknown multipath fading channel. We focus on blind estimation as it does not require any pilot sequences and is therefore applicable to contexts, such as cognitive radio for instance, where little prior signal knowledge is available. The two estimators are respectively based on the time-frequency sparsity of OFDMA signals and on the redundancy induced by the cyclic prefix. Numerical simulations compare the performance of the two algorithms and highlight their complementarity.
\end{abstract}

Index Terms - OFDMA, noise variance, robust estimation, blind estimation.

\section{INTRODUCTION}

Orthogonal Frequency Division Multiple Access (OFDMA) emerges as a promising multiple access technology for new generation wireless networks. It has already been adopted by standards such as WiMAX and 3GPP/LTE and is a candidate for the IEEE 802.22 WRAN. OFDMA systems benefit from scalability, a strong robustness to multipath and a high spectral efficiency.

For such systems, noise variance knowledge can be of prime importance. For instance, it enables propagation channel estimation improvement, it can be needed for signal detection and it is a key decision parameter in adaptive processes such as adaptive modulation and coding (AMC) or adaptive power allocation. In this contribution, we focus on blind estimation as it does not require any pilot sequence and therefore avoids any bandwidth loss. In addition, blind estimation is also of great interest for applications such as sensing for cognitive radio or communication electronic warfare where frame synchronization (operation required to know the pilot locations) is not always possible due to a lack of prior knowledge on the signal.

Usual moment based blind estimators, such as the M2M4 [1] for instance, are not applicable to OFDMA as they require higher order statistics knowledge of the transmitted symbols (this statistics may vary in time and frequency in our context). In this contribution, we investigate different methods to estimate the OFDMA noise variance. These methods are based on distinct signal properties. The first method, "Complex Essential Supremum Estimate (C-ESE)", takes advantage of the possible time-frequency sparsity of the OFDMA signals. In fact, in the cases of low network load, for systems with guard subcarriers and/or for systems using segmentation such as WiMAX for instance, all subcarriers may not be active at all times. C-ESE is a robust estimator based on the recent results in statistical decision and estimation theory developed in $[2,3]$. The second method, purely dedicated to OFDM signals, exploits the redundancy induced by the cyclic prefix (CP). As detailed in the sequel, the two estimation algorithms complement one another as they are suitable to different contexts.

The paper is organized as follows: Section 2 presents the OFDMA signal model. Section 3 recalls the principle of the C-ESE algorithm and details its application to OFDMA. We introduce the second noise variance estimator based on the $\mathrm{CP}$ properties in Section 4. Performance is assessed through simulations in Section 5. Finally, conclusions are presented in Section 6.

\section{OFDMA SIGNAL MODEL}

Assuming that an OFDMA symbol consists of up to $N$ active subcarriers, the discrete-time baseband equivalent transmitted signal is given by ${ }^{1}$

$$
\begin{gathered}
w(m)=\frac{1}{\sqrt{N}} \sum_{k \in \mathbb{Z}} \sum_{n=0}^{N-1} \varepsilon_{k, n} S_{k, n} e^{2 i \pi \frac{n}{N}(m-D-k(N+D))} \\
. g(m-k(N+D)),
\end{gathered}
$$

where $S_{k, n}$ is a sequence of random symbols assumed to be centered, independent and identically distributed (i.i.d). $\varepsilon_{k, n}$ represents a i.i.d sequence of random variables valued in $\{0,1\}$ that express the absence or presence of signal activity in a time-frequency slot $(k, n) . D$ is the cyclic prefix $(\mathrm{CP})$ length and $m \mapsto g(m)$ is the rectangular pulse shaping filter. Let $\{h(\ell)\}_{\ell=0, \cdots, L}$ be a baseband equivalent discrete-time Rayleigh fading channel impulse response of length $L+1$ with $L+1<D$. The received samples of the OFDM signal

\footnotetext{
${ }^{1}$ Note that for noise variance estimation there is no need to differentiate the OFDMA users.
} 
are then expressed as

$$
y(m)=e^{-i\left(2 \pi \delta \frac{m-\tau}{N}+\theta\right)} \sum_{\ell=0}^{L} h(\ell) w(m-\ell-\tau)+x(m),
$$

where $\delta$ is the carrier frequency offset, $\theta$ the initial arbitrary carrier phase, $\tau$ the timing offset and $x(m)$ the additive white Gaussian noise such that $x(m) \sim \mathcal{C N}\left(0, \sigma_{0}^{2}\right)$.

\section{COMPLEX ESSENTIAL SUPREMUM ESTIMATE (C-ESE)}

\subsection{Theoretical results}

The estimator proposed in this section derives from [2, Theorem 1]. This theoretical statistical result, initially motivated by practical issues in radar and speech processing, can read as follows.

Let $Y_{1}, Y_{2}, \ldots, Y_{q}$ be $q$ independent observations that are $d$-dimensional random vectors, each observation $Y_{k}$ being either the sum of some random signal $S_{k}$ and independent noise $X_{k}$ or noise alone. We then have $Y_{k}=\varepsilon_{k} S_{k}+X_{k}$ where $\varepsilon_{k}$ is a random variable valued in $\{0,1\}$ and models the presence or the absence of $S_{k}$. We make the following assumptions: $\varepsilon_{k}, S_{k}$ and $X_{k}$ are independent for every $k=1,2, \ldots, q$; noise is white and Gaussian for each $k=1,2, \ldots, q$ so that $X_{k} \sim \mathcal{N}\left(0, \sigma_{0} \mathbf{I}_{d}\right)$ where $\mathbf{I}_{d}$ stands for the $d \times d$ identity matrix; the signals have finite energy and the probabilities of presence, $\mathrm{P}\left(\left\{\varepsilon_{k}=1\right\}\right)$, of these signals are upper-bounded by some $p \in[0,1)$. The signals are not necessarily i.i.d and their probability distributions are unknown. When the sample size $q$ and the amplitudes of the signals become large, [2, Theorem 1] states the existence of some suitable threshold height $T$ such that the random variable $\sum_{k=1}^{q}\left\|Y_{k}\right\|^{r} I\left(\left\|Y_{k}\right\| \leq \sigma_{0} T\right) / \sum_{k=1}^{q}\left\|Y_{k}\right\|^{s} I\left(\left\|Y_{k}\right\| \leq \sigma_{0} T\right)$ is close to $\sigma_{0}^{r-s} \Upsilon_{r}(T) / \Upsilon_{s}(T)$ where $0 \leq s<r \leq 2, I(A)$ stands for the indicator function of any given event $A$ and $\Upsilon_{\alpha}(x)=\int_{0}^{x} t^{\alpha+\mathrm{d}-1} e^{-t^{2} / 2} \mathrm{~d} t$ for any given $\alpha \in[0, \infty)$ and any $x \in[0, \infty)$. The specific convergence involved in [2, Theorem 1] is omitted here because it will be of no use in the sequel. It is more important to make it clear that the noise standard deviation $\sigma_{0}$ turns out to be the unique positive real value that satisfies this type of convergence. The authors analyse in [2, 3] to what extent the asymptotic conditions involved by this convergence can be relaxed for applications in radar and speech processing where observations are complex values and signals of interest are mostly less present than absent. Therefore, they experimentally address the case where $d=2$, the upper-bound $p$ is $1 / 2, r=1$ and $s=0$, a rather natural choice with respect to the inequalities above that $r$ and $s$ must satisfy. In what follows, we consider the same case to show the potentiality of the method for cognitive radio applications even though the extension to prior probabilities above $1 / 2$ should be addressed in forthcoming work dedicated to OFDMA. When $p \leq 1 / 2, d=2, r=1$ and $s=0$, the experimental results in $[2,3]$ suggest that the asymptotic conditions about the amplitudes of the signals can be relaxed significantly. According to these results, an estimate of the OFDMA noise standard deviation can be computed as follows (see [3] for further details):

[Step 1:] Given $q$ observations $Y_{1}, \ldots, Y_{q}$, compute a first estimate, termed the Essential Supremum Estimate, as a possibly local minimum $\widetilde{\sigma_{0}}$ of the map defined for every positive real value $\sigma$ by

$$
\sup _{\ell \in\{1, \ldots, L\}}\left\{\left|\frac{\sum_{k=1}^{q}\left\|Y_{k}\right\| I\left(\left\|Y_{k}\right\| \leq \beta_{\ell} \sigma \sqrt{2}\right)}{\sum_{k=1}^{q} I\left(\left\|Y_{k}\right\| \leq \beta_{\ell} \sigma \sqrt{2}\right)}-\sigma \Theta\left(\beta_{\ell} \sqrt{2}\right)\right|\right\}
$$

where $L \in \mathbb{N}, \beta_{\ell}=\ell / L$ and $\Theta(x)=\Upsilon_{1}(x) / \Upsilon_{0}(x)=$ $\int_{0}^{x} t^{2} \exp \left(-t^{2} / 2\right) \mathrm{d} t /\left(1-\exp \left(-x^{2} / 2\right)\right)$ for $x \in[0, \infty)$. The reader is asked to refer to $[2,3]$ for the computation of the search interval required for this minimisation in $\sigma$.

[Step 2:] The C-ESE is then

$$
\widehat{\sigma_{0}}=\lambda \sqrt{\frac{\sum_{k=1}^{m}\left\|Y_{k}\right\|^{2} I\left(\left\|Y_{k}\right\| \leq \widetilde{\sigma_{0}} \sqrt{2}\right)}{\sum_{k=1}^{m} I\left(\left\|Y_{k}\right\| \leq \widetilde{\sigma_{0}} \sqrt{2}\right)}}
$$

where $\lambda$ is some constant chosen empirically with respect to the application.

Designed for dealing with signals whose prior probabilities of presence are less than or equal to one half, C-ESE can be regarded as an alternative to the Median Absolute Deviation (MAD) estimator, which performs poorly when the number or the amplitudes of the outliers are too large (see section 5).

\subsection{Application to OFDMA signals}

Let us consider $K$ samples of the received signal $y(m)$. Split this set of observations into $M$ disjoint frames of $N$ samples each such that $K=M N$. Apply an $N$-DFT on each frame. We obtain a matrix $\left[Y_{k, n}\right]_{k \in\{1, \ldots, M\}, n \in\{0, \ldots, N-1\}}$ of complex values where $k$ is the frame index and $n$ the DFT bin number

$$
Y_{k, n}=\frac{1}{\sqrt{N}} \sum_{m=0}^{N-1} y[k N+m] e^{-2 i \pi n m}
$$

For each frame $k$ and each bin $n$, we assume the random presence of an OFDMA frequency component $\tilde{S}_{k, n}$. We therefore have $Y_{k, n}=\tilde{\varepsilon}_{k, n} \tilde{S}_{k, n}+X_{k, n}$. Similarly to Eq. (1), $\tilde{\varepsilon}_{k, n} \in\{0,1\}$ indicates whether the OFDMA frequency component $\tilde{S}_{k, n}$ is present or absent in the $k$ th bin of the $n$th frame. Since the noise is white and Gaussian with standard 
deviation $\sigma_{0}$, the complex random variables $X_{k, n}$ are mutually independent and identically distributed with $X_{k, n} \sim$ $\mathcal{C N}\left(0, \sigma_{0}^{2}\right)$. Note that in the perfect synchronization case and after CP removal $\tilde{\varepsilon}_{k, n}=\varepsilon_{k, n}$ and $\tilde{S}_{k, n}=S_{k, n}$.

Instead of performing an estimate of $\sigma_{0}^{2}$ on the basis of the $M N=K$ values we have, we follow the recommendation of the previous subsection and split our set of observations into subsets of $q$ observations each. We compute an estimate of $\sigma_{0}^{2}$ on each subset with $d=2$ and then average all the ${\widehat{\sigma_{0}}}^{2}$ obtained. In order to deal with $q$ observations that can reasonably be considered as mutually independent, these observations can be chosen randomly amongst the $M N$ values we have.

\subsection{Discussion}

The C-ESE is a very powerful noise variance estimation method as it is based on very few priors. In fact, it only considers that the signal is sparse and that the noise is white and Gaussian and assumes mutual independency between the different random variables. No assumption is made on the signal distribution. However, further study of the C-ESE is required to get better insight into the behaviour of these estimates, in particular with regard to the constant $\lambda$ in Eq. (2). In addition, theoretical results in [2] are established for any sparsity degree but at this stage of investigation C-ESE implementation is limited to the case where the signal is less present than absent which is not always verified for OFDMA signals.

\section{CYCLIC PREFIX REDUNDANCY BASED ALGORITHM}

\subsection{Algorithm}

As in [4], we here suggest to take advantage of the OFDM signal particular structure to estimate the noise variance. More precisely, we show hereafter that the noise variance can be estimated thanks to the redundancy induced by the $\mathrm{CP}$. In fact, the $\mathrm{CP}$ use leads to $x(k(N+D)+m)=$ $x(k(N+D)+N+m)$, for any integer $k$ and any $m \in$ $\{0, \cdots, D-1\}$. It is then straightforward to see that if we assume perfect synchronization ${ }^{2}$ at reception (i.e $\tau=0$ and $\delta=0$ ) and a time-invariant channel over an OFDM symbol duration, we can get $D-L$ noise variance estimates defined as

$$
\breve{\sigma}_{0, u}^{2}=J(u), L \leq u \leq D-1
$$

with

$$
\begin{aligned}
J(u)=\frac{1}{2 M(D-u)} \sum_{k=0}^{M-1} \sum_{m=u}^{D-1} \mid y(k(N+D)+m)- \\
\left.y(k(N+D)+N+m)\right|^{2}
\end{aligned}
$$

\footnotetext{
${ }^{2}$ for NDA OFDM synchronization algorithms refer to [5] and [6]
}

where $M$ denotes the number of OFDM symbols in the observation window. It can be easily shown that the estimator with the smallest variance is found for $u=L$. The difficulty is then to estimate $L$. Cui et al. suggested an estimator in [7] but it has the major disadvantage of being based on a threshold level chosen arbitrarily. To overcome this limitation we hereafter propose a method inspired by maximum likelihood estimation.

From Eq. (3), $J(u)$ can be expressed as

$$
J(u)=\left(1-\frac{1}{D-u}\right) J(u+1)+\xi(u)
$$

where $\xi(u)$ is a random variable that verifies, for $L \leq u \leq$ $D-1$,

$$
\frac{2 M(D-u)}{\sigma_{0}^{2}} \xi(u) \sim \chi_{2 M}^{2},
$$

where $\chi_{2 M}^{2}$ denotes the chi-square distribution with $2 M$ degrees of freedom. $L$ is then estimated using the likelihood function $f\left(\mathbf{X}_{u} \mid L=u\right)$ with $\mathbf{X}_{u}$ the multivariate observation variables defined as $\mathbf{X}_{u}=(\xi(u), \xi(u+1), \cdots, \xi(D-1))$. The different $\xi(u)$ being independent, $\hat{L}$ is given by

$$
\begin{aligned}
& \hat{L}=\underset{u}{\operatorname{argmax}}\left[\prod_{m=u}^{D-1} f(\xi(m) \mid L=u)\right]^{1 /(D-u)}, \\
& 0 \leq u \leq D-1
\end{aligned}
$$

where $f(\xi(m) \mid L=u)$ is computed thanks to Eq. (4) by making the approximation that $\sigma_{0}^{2} \simeq J(u)$. Note that because the observations $\mathbf{X}_{u}$ are of variable lengths, Eq. (5) is defined as an average likelihood which is the geometric mean of the individual likelihood elements.

\subsection{Discussion}

The CP based algorithm has the major advantage of not being dependent on the subcarrier allocation rate as it exploits only OFDM (and no OFDMA) signal properties. Moreover, in contrast with non data-aided estimator such as [7], it is not based on the subjective choice of a threshold level. However, compared to the C-ESE method, the estimator derived in this section suffers from the drawbacks of requiring prior time and frequency synchronization and is dependent on the $\mathrm{CP}$ and channel impulse response durations.

\section{SIMULATIONS}

In the following, all the results are averaged over 1000 Monte Carlo runs. We consider 512-subcarrier OFDMA systems with $D=128$. The slot allocation is assumed to be i.i.d. The number of OFDMA symbols available at reception is set to 25. The Signal-to-Noise Ratio (SNR) is defined as $\mathrm{SNR}(\mathrm{dB})=10 \log _{10}\left(\mathbb{E}\left[\left|\varepsilon_{k, n} S_{k, n}\right|^{2}\right] / \sigma_{0}^{2}\right)$. The simulated propagation channel is a time-invariant discrete-time channel 


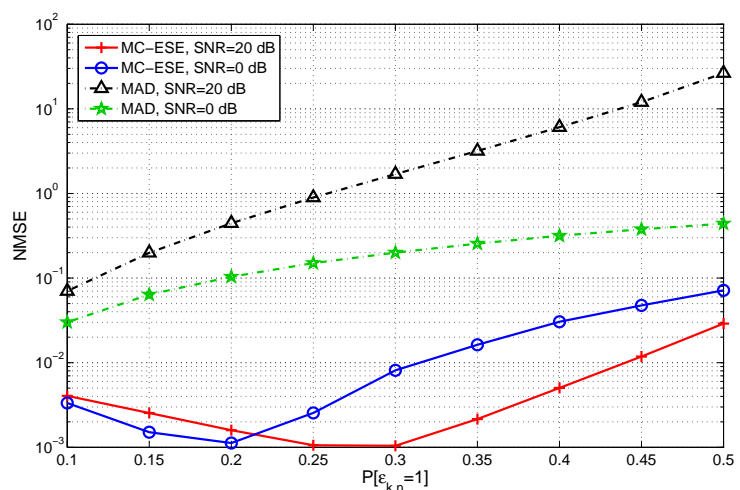

Fig. 1. MAD and C-ESE performance comparison $(L=$ $0.9 D)$.

$\left\{h_{k}(\ell)\right\}_{\ell=0, \cdots, L}$ with an exponential decay profile for its nonnull component (i.e., $\mathbb{E}\left[\left|h_{k}(\ell)\right|^{2}\right]=G e^{-\ell / \mu}$ for $\ell=0, \cdots, L$ and $G$ is chosen such that $\left.\sum_{\ell=0}^{L} \mathbb{E}\left[\left|h_{k}(\ell)\right|^{2}\right]=1\right) . \mu$ is set to 32.

Figure 1 compares the Normalized Mean Square Error (NMSE) of the C-ESE with the NMSE of the well known MAD estimates. $\lambda$ is set to $\sqrt{2}$. We observe that the performance of each estimator strongly depends on $\mathrm{P}\left(\left\{\varepsilon_{k, n}=1\right\}\right)$. Moreover, C-ESE clearly outperforms the MAD method as the latter is not resistant to large outlier numbers or amplitudes.

The CP based algorithm performance is shown in Figure 2. As expected, it indicates that the channel length has a strong influence on the estimation results. In addition, it can be seen that the performance gets better as the SNR increases. At low SNR, the estimator defined in Eq. (5) occasionally under-estimates $L$ which leads to performance degradation.

As explained previously and illustrated in Figure 1 and 2, the two proposed methods may be applicable in different contexts as they rely on different parameters. For instance, when $L \ll D$ is verified, the use of the CP based method is totally relevant whereas C-ESE is suitable for situations where $\mathrm{P}\left(\left\{\varepsilon_{k, n}=1\right\}\right)$ is low as it performs well in these cases and does not rely on prior synchronization.

\section{CONCLUSIONS AND PERSPECTIVES}

In this contribution, we have suggested to take advantage of the sparse nature of the OFDMA signals as well as the redundancy induced by the cyclic prefix to derive two new blind noise variance estimators for OFDMA signals. These two estimators can be considered as complementary as they are relevant in different contexts. C-ESE appears to be a very promising algorithm as it requires very little signal prior knowledge and yields good performance. Forthcoming work will involve further exploration of the theoretical results developed in [2] in order to extend the C-ESE estimator to cases where prior

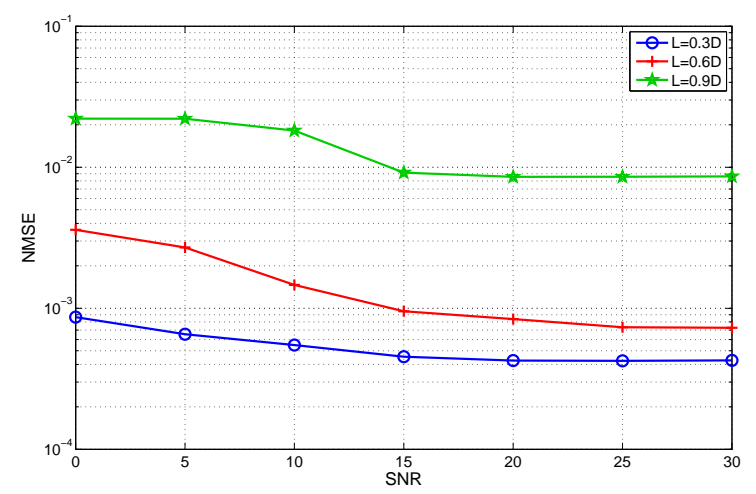

Fig. 2. NMSE of the $\mathrm{CP}$ based noise variance estimator $\left(\mathrm{P}\left(\left\{\varepsilon_{k, n}=1\right\}\right)=0.5\right)$.

probabilities of presence of OFDMA signals can be above 0.5. Regarding the CP based method, simulations exhibit excellent results. Synchronization impairments are currently under evaluation.

\section{REFERENCES}

[1] T.R. Benedict and T.T.Soong, "The Joint Estimation of Signal and Noise from the Sum Envelope," IEEE Transactions on Information Theory, vol. 13, no. 3, pp. 447454, Jul. 1967.

[2] D. Pastor, "A theoretical result for processing signals that have unknown distributions and priors in white Gaussian noise," Computational statistics and data analysis, vol. 52, pp. 3167-3186, 2008.

[3] D. Pastor and A. Amehraye, "Algorithms and Applications for Estimating the Standard Deviation of AWGN when Observations are not Signal-Free," Journal of Computers, vol. 2, no. 7, Sept. 2007.

[4] F-X Socheleau, A. Aissa-El-Bey, and S. Houcke, "Non Data-Aided SNR Estimation of OFDM Signals," IEEE Communications Letters, vol. 12, no. 11, Nov. 2008.

[5] J. van de Beek, M. Sandell, and P.O. Borjesson, "ML estimation of time and frequency offset in OFDM systems," IEEE Trans. on Signal Processing, vol. 49, pp. 1800-1805, Jul. 1997.

[6] B. Park, E. Ko, H. Cheon, C. Kang, and D. Hong, “A blind OFDM synchronization algorithm based on cyclic correlation," in IEEE Globecom Conf., Nov. 2001, vol. 5, pp. 3116-3119.

[7] T. Cui and C. Tellambura, "Power delay profile and noise variance estimation for OFDM," IEEE Communications Letters, vol. 10, no. 1, Jan. 2006. 